\title{
A two mixture fraction flamelet model for large eddy simulation of turbulent flames with inhomogeneous inlets
}

\author{
Bruce A. Perry ${ }^{\mathrm{a}, *}$, Michael E. Mueller ${ }^{\mathrm{a}}$, Assaad R. Masri ${ }^{\mathrm{b}}$ \\ ${ }^{a}$ Department of Mechanical and Aerospace Engineering, Princeton University, USA \\ ${ }^{b}$ School of Aerospace, Mechanical, and Mechatronic Engineering, The University of Sydney, Australia
}

\begin{abstract}
A revised flamelet/progress variable (FPV) model in which two mixture fractions are defined has been developed to address the limitations of single mixture fraction FPV models that presume a single, compositionally uniform fuel stream. The revised model is applied in Large Eddy Simulation of the modified University of Sydney turbulent jet burner with compositionally inhomogeneous inlet conditions. The first mixture fraction $(Z)$ characterizes the mixing between the methane/air mixture issuing from the burner and the surrounding coflow air. The second mixture fraction $\left(Z^{*}\right)$ tracks mixing of methane and air without regard to the point of origin of the air. Additionally, a fuel premixing fraction $(F)$ has been defined that corresponds to the fuel side boundary condition for the solution of the 1D flamelet equations in terms of the first mixture fraction. Two methods of characterizing the thermochemical state are considered, one using $Z$ and $Z^{*}$ and the other using $Z$ and $F$. Comparison of temperature and species predictions with experimental data shows that the structure of the flame with inhomogeneous inlets cannot be predicted by a single mixture fraction model while this is successfully achieved with the two mixture fraction models. Even for a near-homogeneous inlet with only minor variations in composition, the two mixture fraction model predictions provide significant improvements over a single mixture fraction model.
\end{abstract}

Keywords: Turbulent combustion, Flamelet modeling, Large eddy simulation, Inhomogeneous jet

\section{Introduction}

A common approach to turbulent combustion simulation that balances accuracy and cost considerations is Large Eddy Simulation (LES) coupled with reduced order models for combustion chemistry. Flamelet models, which make assumptions about the flame structure to decrease the dimensionality of the thermochemical state space, have been successfully applied to a range of nonpremixed and premixed combustion systems [1]. Traditional nonpremixed flamelet models are based on a coordinate transformation to 1D mixture fraction space, where mixture fraction is a parameter that characterizes the mixing between fuel and oxidizer streams. The definition of mixture fraction limits the range of applicability of these models by requiring that there be only two distinct, compositionally homogeneous inlets.

There are many practical combustion systems and laboratory burners that have more complex boundary conditions than allowed by models using a single mixture fraction. Diesel engines that utilize split injection strategies were shown to have lower soot emissions than those using a single injection [2]. These systems must be treated as having multiple inlets in order to capture the interactions between the different injection events. Exhaust gas recirculation can also add a third stream in addition

*Corresponding author

Email address: baperry@princeton.edu (Bruce A. Perry) 
to fuel and oxidizer. Similarly, the jet-in-hot-coflow (JHC) burner of Dally et al. [3] is a three-stream system, consisting of a central fuel jet, a hot coflow generated by mixing the exhaust of a secondary burner with air and nitrogen, and a cool air coflow. In addition, the pilot of any piloted jet burner is a third inlet stream, even if in most cases it can reasonably be modeled as a mixture of the fuel and oxidizer streams.

To address these limitations and broaden the range of applicability of the flamelet approach, more advanced models have been developed that can account for a third inlet stream by defining a second mixture fraction. These models require solving the flamelet equations in the resulting 2D mixture fraction space and were successfully applied to the simulation of split injection diesel engines [4, 5]. If appropriate conditions hold, the 2D flamelet equations can be reduced back to the original 1D equations but with variable boundary conditions that are specified by the second mixture fraction. This approach has been employed for LES of the JHC burner [6, 7].

Combustion systems with inhomogeneous inlets represent a similar challenge for traditional flamelet models. These systems are practically important and quite common because achieving perfect mixing is often unrealistic or even undesirable. For example, in some gas turbine burners the fuel stream is partially premixed with air, and significant inhomogeneity in this mixture plays a key role in the flame dynamics [8, 9]. Meares et al. [10, 11] have recently shown that combustion of inhomogeneous fuel/air mixtures can significantly enhance the stability of laboratory-scale turbulent jet flames. The burner used in these studies is similar to the established Sydney piloted jet burner [12], which consists of a central fuel jet surrounded by a pilot annulus, but an additional concentric tube has been added within the central jet, allowing for separate fuel and air streams. This tube can be retracted, resulting in a controlled amount of mixing between fuel and air upstream of the nozzle. Experimental observations indicate that the nonpremixed combustion mode dominates throughout most of the flame, but stratified-premixed combustion may occur in the small region immediately downstream of the nozzle when there is inhomogeneous mixing [11, 13]; this is hypothesized to be the mechanism for increased stability.

In this paper, a two mixture fraction flamelet model for an inhomogeneous fuel stream is developed and applied in LES of the modified Sydney burner, which is described in detail with related measurements in Section 2 In Section 3 two formulations of the two mixture fraction model are presented. Based on the previous experimental observations, a nonpremixed approach is used. The predictions of both formulations of the two mixture fraction model are compared to predictions using a traditional flamelet model and experimental data in Section 4 Two cases are considered: a flame where the jet inlet is nearly compositionally homogeneous and a flame where the jet inlet is highly inhomogeneous. The first objective of this comparison is to evaluate the strengths and weaknesses of both two mixture fraction approaches for modeling combustion with an inhomogeneous fuel stream. The second objective is to illuminate the effects of inhomogeneity on the flame structure.

\section{Burner configuration and measurements}

The modified burner assembly that generates inhomogeneous inlet conditions is described in detail by Meares et al. [10]. It consists of two outer tubes forming the pilot annulus and a retractable central tube that controls mixing between fuel and air in the jet. The inner diameters of the three tubes are $4 \mathrm{~mm}, 7.5 \mathrm{~mm}$, and $18 \mathrm{~mm}$, and the wall thicknesses are $0.25 \mathrm{~mm}, 0.25 \mathrm{~mm}$, and $0.2 \mathrm{~mm}$, respectively. The jet diameter $\left(D_{J}\right)$ is defined as the inner diameter of the middle tube (referred to hereon as the 'mixing tube') from which the fuel/air mixture issues. A wind tunnel provides coflowing air at $15 \mathrm{~m} / \mathrm{s}$. The turbulent jet flame from this burner has been characterized extensively at the University of Sydney [10] and the Combustion Research Facility at Sandia National Laboratory [11, 13]. Composition and temperature at several axial locations for methane fuel were measured 
at Sandia using Raman-Rayleigh scattering and CO-laser induced fluorescence (LIF) imaging. The cases studied here use a five-gas pilot (5GP) consisting of $\mathrm{C}_{2} \mathrm{H}_{2}, \mathrm{H}_{2}, \mathrm{CO}_{2}, \mathrm{~N}_{2}$, and air, which matches the adiabatic flame temperature and equilibrium composition of a stoichiometric $\mathrm{CH}_{4} /$ air mixture.

The recession distance $\left(L_{r}\right)$ of the central tube from the nozzle exit plane can be varied from 0 to $300 \mathrm{~mm}$. When $L_{r}=0$, the burner is functionally identical to a typical nonpremixed burner. High values of $L_{r}$ allow for nearly complete mixing and homogeneous inlet conditions similar to the well-documented Sandia flame series [14], but intermediate values of $L_{r}$ result in incomplete mixing and therefore inhomogeneous boundary conditions. At intermediate recession distances, a substantial increase in blowoff velocity relative to the nonpremixed and homogeneous limits was observed for the configuration with fuel in the retractable central tube and air in the surrounding annulus. The stability increase was the largest for $L_{r}=75 \mathrm{~mm}$ using a volumetric air to fuel ratio of 2.0. This case and the corresponding essentially homogeneous inlet $\left(L_{r}=300 \mathrm{~mm}\right)$ case were selected for simulation. The homogeneous and inhomogeneous cases were run with bulk jet velocities $\left(U_{J}\right)$ of $59 \mathrm{~m} / \mathrm{s}(70 \%$ of blow off) and $57 \mathrm{~m} / \mathrm{s}$ (50\% of blow off), corresponding to jet Reynolds numbers of 27,800 and 26,800, respectively. The full names for these two cases used by Barlow et al. [13] and in the online data set are FJ200-5GP-Lr300-59 and FJ200-5GP-Lr7557, but these names are subsequently abbreviated to Lr300 and Lr75 in this paper.

\section{Modeling framework}

\subsection{Two mixture fraction flamelet model}

Flamelet modeling is a well-established reduced-order method to determine the thermochemical state in turbulent nonpremixed combustion simulations [15, 16]. In these models, mixing between compositionally homogeneous fuel and oxidizer streams is typically characterized through a single parameter, the mixture fraction $(Z)$, which is defined by the equation [17]:

$$
\frac{\partial}{\partial t}(\rho Z)+\frac{\partial}{\partial x_{j}}\left(\rho u_{j} Z\right)=\frac{\partial}{\partial x_{j}}\left(\rho D_{Z} \frac{\partial Z}{\partial x_{j}}\right),
$$

where $\rho$ is the density and $D_{Z}$ is the mixture fraction diffusivity, with the boundary conditions that $Z=1$ in the fuel inlet $\left(B^{F}\right)$ and $Z=0$ in the oxidizer inlet $\left(B^{O}\right)$.

Under the assumption that gradients in the flame-normal $(Z)$ direction dominate, the 3D governing equations in physical space can be transformed into $1 \mathrm{D}$ equations in $Z$ space, for example:

$$
-\rho \frac{\chi Z}{2} \frac{\partial^{2} Y_{i}}{\partial Z^{2}}=\dot{m}_{i}
$$

where $Y_{i}$ is the mass fraction of species $i, \dot{m}_{i}$ is the correpsonding reaction source term, and $\chi_{Z}$ is the scalar dissipation rate, defined as $\chi_{Z}=2 D_{Z} \nabla Z \cdot \nabla Z$. This form of the equation assumes no unsteady effects and unity Lewis numbers. The boundary conditions are given by the conditions at $B^{O}$ for $Z=0$ and the conditions at $B^{F}$ for $Z=1$. The flamelet equations are solved for a range of reference values of scalar dissipation rate using a functional form for $\chi_{Z}(Z)$ based on a $1 \mathrm{D}$ counterflow diffusion flame [15]. In the flamelet/progress variable (FPV) approach [18] used in this work, flamelet solutions are then reparameterized using a progress variable, $\mathrm{C}=\mathrm{Y}_{\mathrm{H}_{2} \mathrm{O}}+\mathrm{Y}_{\mathrm{H}_{2}}+Y_{\mathrm{CO}_{2}}+Y_{\mathrm{CO}}$ [19].

For the case of an inhomogeneous inlet stream (taken to be the fuel stream without loss of generality), a second mixture fraction $\left(Z^{*}\right)$ is required to characterize the mixing state of the system. The second mixture fraction is defined by an equation identical to Eq. (1) but with the boundary conditions $Z^{*}\left(B^{O}\right)=0$ and $Z^{*}\left(B^{F}\right)=Y_{F}(\mathbf{x}, t)$, where $Y_{F}(\mathbf{x}, t)$ at the fuel inlet must be specified. For the Sydney burner, assuming no reaction upstream of the nozzle, this is equivalent to extending the domain of 


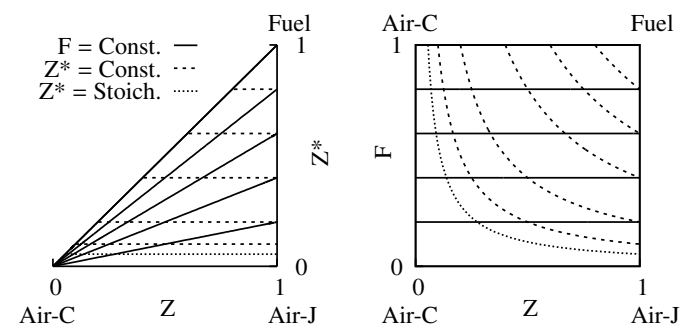

Figure 1: Two-dimensional state space for mixing between fuel in the jet, air in the jet (Air-J), and air in the coflow (Air-C) parametrized by $\left(Z, Z^{*}\right)$ and $(Z, F)$.

simulation to include the mixing tube and solving the $Z$ and $Z^{*}$ transport equations with the boundary conditions $Z\left(B^{O}\right)=0$, $Z\left(B^{F, 1}\right)=Z\left(B^{F, 2}\right)=1, Z^{*}\left(B^{O}\right)=Z^{*}\left(B^{F, 1}\right)=0$, and $Z^{*}\left(B^{F, 2}\right)=1$, where $B^{F, 1}$ corresponds to the air annulus entering the mixing tube and $B^{F, 2}$ corresponds to the fuel stream entering the mixing tube. The two mixture fractions are defined in this manner because it results in clear and distinct physical interpretations: $Z$ characterizes the mixing between the coflow and the fuel/air mixture issuing from the mixing tube, while $Z^{*}$ indicates the local availability of fuel. For homogeneous inlets, these are equivalent and $Z=Z^{*}$.

It is also useful to define an additional parameter, the fuel premixing fraction, $F \equiv Z^{*} / Z$, which represents the local fuel availability considering only mass that originated in the jet rather than the coflow. Higher values of $F$ indicate less premixing with air from the central annulus, independent of the extent of mixing between the jet and coflow. A transport equation for $F$ can be derived from its definition and the transport equations that define $Z$ and $Z^{*}$ :

$$
\frac{\partial}{\partial t}(\rho F)+\frac{\partial}{\partial x_{j}}\left(\rho u_{j} F\right)=\frac{\partial}{\partial x_{j}}\left(\rho D_{Z} \frac{\partial F}{\partial x_{j}}\right)+\frac{\rho \chi_{F Z}}{Z},
$$

where $\chi_{F Z}=2 D_{Z} \nabla Z \cdot \nabla F$ and it has been assumed that $D_{Z^{*}}=D_{Z}$.

Hasse and Peters used a three-scale asymptotic analysis to derive 2D flamelet equations for systems that require two mixture fractions [4]. Their analysis assumes variations of both mixture fractions occur in overlapping regions of physical space and have similar characteristic length scales. For the Sydney burner with inhomogeneous inlets, the partial premixing of fuel and air occurs upstream of the nozzle, so the largest variations of $F$ occur in the mixing tube and in the center of the jet just downstream from the nozzle, while $Z$ is uniform within the mixing tube but varies in the mixing layer between the jet and pilot/coflow. Therefore, rather than solving the 2D flamelet equations, the thermochemical state is modeled using solutions to Eq. (2) with a variable boundary condition on the fuel side that depends on the fuel premixing fraction: $Y_{i}\left(B^{F}\right)=Y_{i}(F)$.

The state space for mixing between the the inhomogeneous jet and the air coflow is two-dimensional and can be parametrized by $\left(Z, Z^{*}\right)$ or $(Z, F)$ as shown in Fig. 1] $\left(Z, Z^{*}\right)$ space is triangular because $Z \geq Z^{*}$ at all boundaries, and thus for equal diffusivities $Z \geq Z^{*}$ everywhere in the domain. In contrast, $(Z, F)$ space is square, but there is a singularity at $Z=0$ where all values of $F$ correspond to the same state (coflow air) due to the definition $F \equiv Z^{*} / Z$. On these diagrams, lines of constant $F$ correspond to the 1D flamelet solutions that are used to populate the state space with thermochemical data. Because the full 2D flamelet equations are not considered, there is no interaction between flamelets at different values of $F$. The full state space has a third dimension, $C$, to account for all reacting states, and a family of flamelet solutions for each value of $F$ is required to populate this 3D space with thermochemical state data. Therefore, the thermochemical state of the system can be determined if $Z, C$, and either $Z^{*}$ or $F$ are specified. These two parameterizations are both examined and are subsequently refered to as the $\left(Z, Z^{*}\right)$ and $(Z, F)$ approaches. 


\subsection{Equation closure for LES}

In LES, filtered values of the scalars are determined using equations of the form

$$
\frac{\partial}{\partial t}(\bar{\rho} \widetilde{\psi})+\frac{\partial}{\partial x_{j}}\left(\bar{\rho} \widetilde{u}_{j} \widetilde{\psi}\right)=\frac{\partial}{\partial x_{j}}\left(\bar{\rho} \widetilde{D}_{\psi} \frac{\partial \widetilde{\psi}}{\partial \alpha_{j}}\right)+\frac{\partial}{\partial x_{j}}\left(\bar{\rho} \widetilde{u}_{j} \widetilde{\psi}-\bar{\rho} \widetilde{u}_{j} \psi\right)+\bar{S}_{\psi},
$$

where $\widetilde{\psi}$ is the density-weighted filtered value of the scalar and $\bar{S}_{\psi}$ is the corresponding filtered source term. All subfilter stresses and fluxes are calculated using dynamic Smagorinski-like models [20, 21]. In addition to the scalars required to parametrize the thermochemical state, an equation must also be solved for $\widetilde{Z^{2}}$ to determine the subfilter variance for $Z$. In vector form, for $\psi=\left[\widetilde{Z}, \widetilde{Z^{2}}, \widetilde{Z^{*}}, \widetilde{F}, \widetilde{C}\right]^{T}$,

$$
\overline{\dot{S}}=\left[0, \bar{\rho} \widetilde{\chi}_{Z}, 0, \bar{\rho} \overline{\left(\frac{\chi_{F Z}}{Z}\right)}, \overline{\dot{m}}_{C}\right]^{T},
$$

where all nonzero source terms are unclosed and need to be modeled.

The filtered chemical source term for $C$ is modeled using a presumed subfilter probability distribution function (PDF) approach, shown for the $(Z, F)$ parametrization:

$$
\overline{\dot{m}}_{c}=\bar{\rho} \iiint \frac{1}{\rho} \dot{m}_{c}(Z, F, C) \widetilde{P}(Z, F, C) d Z d F d C,
$$

where $\dot{m}_{c}$ is obtained from flamelet solutions and $\widetilde{P}$ is the presumed subfilter PDF. The subfilter PDF for $Z$ is taken to be a $\beta$-distribution with mean $\widetilde{Z}$ and variance $Z_{v}=\widetilde{Z^{2}}-\widetilde{Z}^{2}[18,19]$. Single flamelet closure is assumed for the progress variable [18]. A similar single flamelet closure approach is used to model the subfilter conditional PDFs for $Z^{*}$ and $F$ :

$$
\begin{aligned}
\widetilde{P}(F \mid Z) & =\delta(F-\widetilde{F}) . \\
\widetilde{P}\left(Z^{*} \mid Z\right) & =\delta\left(Z^{*} / Z-\widetilde{Z^{*}} / \widetilde{Z}\right) .
\end{aligned}
$$

These PDFs imply that for both approaches the convolution in Eq. 66 is carried out on lines of constant $F$ along a single

flamelet solution. For the case of two partially premixed streams mixing into a third stream, DNS studies [22] indicate that this three-parameter PDF would be far more accurate than the three-parameter Dirichlet distribution used in other works [4, 23]. The 1D flamelet solutions are calculated and convoluted with the presumed PDFs a priori to generate a 4D lookup table of thermochemical state information parameterized by $\left(\widetilde{Z}, Z_{v}, \widetilde{Z^{*}}, \widetilde{C}\right)$ or $\left(\widetilde{Z}, Z_{v}, \widetilde{F}, \widetilde{C}\right)$.

The filtered scalar dissipation rate $\widetilde{\chi}_{Z}$ is decomposed into resolved and subfilter parts, and the latter is modeled using an established linear relaxation model:

$$
\widetilde{\chi}_{Z}=2 \widetilde{D}_{z} \nabla \widetilde{Z} \cdot \nabla \widetilde{Z}+C_{\chi} \frac{v_{t}}{\Delta^{2}}\left(\widetilde{Z^{2}}-\widetilde{Z}^{2}\right)
$$

where $C_{\chi}$ is taken to be 20, $v_{t}$ is the turbulent diffusivity, and $\Delta$ is the filter width [24]. The filtered source term in the $\widetilde{F}$ equation can be closed using an analgous model for the subfilter cross-dissipation and neglecting the subfilter correlation between $Z$ and $\chi_{F Z}$ :

$$
\overline{\left(\frac{\chi F Z}{Z}\right)}=\frac{1}{\widetilde{Z}}\left(2 \widetilde{D}_{Z} \nabla \widetilde{Z} \cdot \nabla \widetilde{F}+C_{\chi} \frac{v_{t}}{\Delta^{2}}(\widetilde{F Z}-\widetilde{F} \widetilde{Z})\right) .
$$

Because $\dot{S}_{F}$ should be zero when $Z=0$ since $\nabla Z=0$ and $\nabla F=0, \bar{S}_{F}$ is set to zero when $\widetilde{Z}$ is below a threshold value.

Although the subfilter cross-dissipation was neglected in previous works using similar models [7], it was found to be necessary in this case and neglecting it leads to discrepancies with both the experimental data and the $\left(Z, Z^{*}\right)$ approach.

Since $\widetilde{F Z}=\widetilde{Z^{*}}$, it is necessary to solve an equation for $\widetilde{Z^{*}}$ to close the $\widetilde{F}$ equation source term and five scalar equations are required for the $(Z, F)$ approach as opposed to four for the $\left(Z, Z^{*}\right)$ approach. This leads to an apparent inconsistency with 
the presumed PDF form, which implies that $\widetilde{Z^{*}}=\widetilde{F} \widetilde{Z}$. To obtain a nonzero covariance of $F$ and $Z$, a five-parameter bivariate $\beta$-distribution [22] would be needed, but the computer memory requirements for pretabulation with such a PDF would be intractable. However, the inconsistency is minor because the covariance of $F$ and $Z$ is typically quite small. The $F$ source term only becomes significant at the fringes of the jet where the cross-dissipation is amplified since $Z$ takes a small but nonzero value. Additionally, there is no consistency issue for the $\left(Z, Z^{*}\right)$ approach because it is not necessary to use the model in Eq. (9).

\subsection{Implementation}

The simulations presented in this work use the combustion models described above as implemented in NGA, a structured finite difference LES code for low Mach number turbulent reacting flows based on the numerical methods of Desjardins $e t$ al. [25]. Second-order spatial operators are used to discretize the momentum and continuity equations. Scalar equations are discretized using a third-order weighted essentially non-oscillatory (WENO) scheme [26]. For construction of the flamelet library, FlameMaster [27] is used to generate solutions of the steady flamelet equation based on the GRI-3.0 mechanism [28]. The fuel is assumed to be pure methane.

The domain for the flame simulation extends $50 D_{J}$ downstream and is discretized using stretched grid with 256,128 , and 64 points in the $x, r$, and $\theta$ directions. Conditional statistics at several axial locations downstream were collected over a period of approximately $10 \mathrm{~ms}\left(\sim 76 D_{J} / U_{J}\right)$, which was sufficient to achieve good statistical convergence. Plug flow inlet conditions are assumed for the air coflow and pilot. For the $(Z, F)$ approach, the coflow inlet boundary condition is not well defined, as $F=Z / Z^{*}=0 / 0$ in the coflow. However, it was found that the predictions are not sensitive to the value of $F$ in the coflow, which was taken to be zero for the results discussed here. The pilot is modeled as a stoichiometric methane/air mixture $\left(Z^{*}=0.055\right)$ with an elevated progress variable indicating a reacted state and an inlet velocity specified to match the experimental pilot mass flow rate. Additionally, the value for either $F$ or $Z$ in the pilot must be specified but is not fixed by physical constraints. Here, the value of $F$ is set to match the average value in the central jet, $F=0.205$, but the model is not strongly sensitive to this value.

Two non-reacting simulations of the flow upstream of the jet exit plane are performed to generate the inhomogeneous inlet boundary condition for the main simulation of the jet flame. The first simulation is a short pipe/annulus flow with periodic boundary conditions to generate a fully developed flow. The second simulation uses this profile as the inflow condition to the $75 \mathrm{~mm}$ or $300 \mathrm{~mm}$ zone of the mixing tube. By definition, $Z=1$ at the jet exit plane, but the boundary conditions for $F, Z^{*}$, and velocity are functions of space and time that are specified from the outflow conditions of the mixing tube. This domain is discretized with either 179, 160, and 64 grid points (Lr75 case) or 500,160, and 64 grid points (Lr300 case) in the $x, r$, and $\theta$ directions, respectively.

\section{Results and discussion}

In this section, LES flame structure predictions using both two mixture fraction approaches as well as a single mixture fraction model are compared to experimental measurements. For the single mixture fraction model, the fuel-side boundary condition for the flamelet equations is taken to be $Y_{F}\left(B^{F}\right)=1$ to ensure that $Z \leq 1$ everywhere in the domain (and thus $Z$ and $Z^{*}$ are equivalent for this model). Since the primary objective here is to compare the combustion models, statistics conditioned on $Z^{*}$ are compared to isolate the effects of the combustion models and minimize the impact of turbulence models. Statistics 

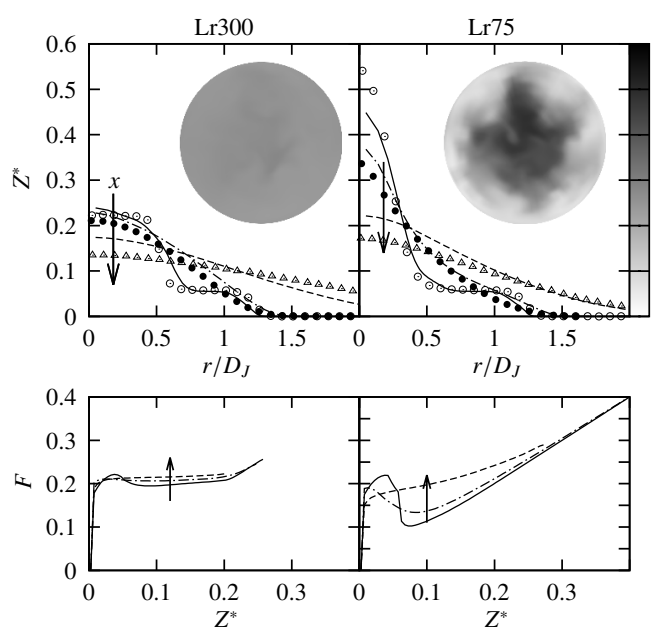

Figure 2: Experimental measurements (points) and $(Z, F)$ model predictions (lines) for the mean radial mixture fraction profiles at $x / D_{J}=1,10$, and 30 are shown in the top row. The arrows indicate increasing distance downstream. The overlays show predictions of the instantaneous mixture fraction distribution at the nozzle exit plane from the simulations of the mixing tube. The conditional mean value of the fuel premixing fraction $(F)$ at the same three axial locations is plotted in the bottom row. The LES results correspond to statistics of the resolved quantities.

are conditioned on $Z^{*}$ rather than $Z$ because air originating from the jet is compositionally identical to air originating from the coflow, so $Z$ cannot be determined from the experimental data.

\subsection{Mean flame structure}

The top row of Fig. 2 shows how the mixture fraction corresponding to fuel content $\left(Z^{*}\right)$ varies downstream of the nozzle, using both experimental measurements and LES predictions. Although simulation results are only plotted for the $(Z, F)$ model, the $Z^{*}$ profiles in physical space are largely unaffected by the combustion model. Close to the nozzle $\left(x / D_{J}=1\right)$, the mixture fraction profile for the Lr300 case is nearly uniform within the jet $\left(r / D_{J} \leq 0.5\right)$ as expected for this near-homogeneous case. In contrast, for the Lr75 case significant inhomogeneity is apparent, with the value of $Z^{*}$ being very rich $(\sim 0.6)$ near the centerline but close to the stoichiometric value at the edge of the jet. This is further demonstrated by the instantaneous distributions of $Z^{*}$ at the nozzle predicted by the simulations of the Lr75 and Lr300 mixing tubes. Moving downstream, the spreading of the jet is captured qualitatively by the simulations but slightly underpedicted quantitatively.

The profiles of $F$ conditioned on $Z^{*}$ at the same three axial locations are plotted in the bottom row of Fig. 2 . For the Lr300 case, there are minor variations in $F$ near the nozzle, but mixing within the jet quickly eliminates these variations and $F$ becomes constant across all values of $Z^{*}$. In contrast, for the Lr75 case, the value of $F$ changes significantly within the flame. These variations diminish as mixing occurs in the jet downstream of the nozzle, indicating that the effects of inhomogeneity decrease going downstream. Note that the boundary condition in the coflow requires that $F=0$ when $Z^{*}=0$, but this condition does not affect the value of $F$ in the bulk of the flame.

The conditional temperature profiles at three axial locations in the Lr300 flame are shown in the top left row of Fig. 3 . On the lean side of the flame, all combustion models accurately predict temperature at all axial locations. The lean side of the 1D flamelet solutions is minimally affected by the fuel-side boundary condition, so an additional parameter to characterize the fuel/air premixing is not required to determine the thermochemical state for these mixture fractions.

On the rich side of the flame, significant differences between the combustion models are observed, with the two mixture fraction models providing substantially improved agreement with the experiment. Near the nozzle, the discrepancy between 

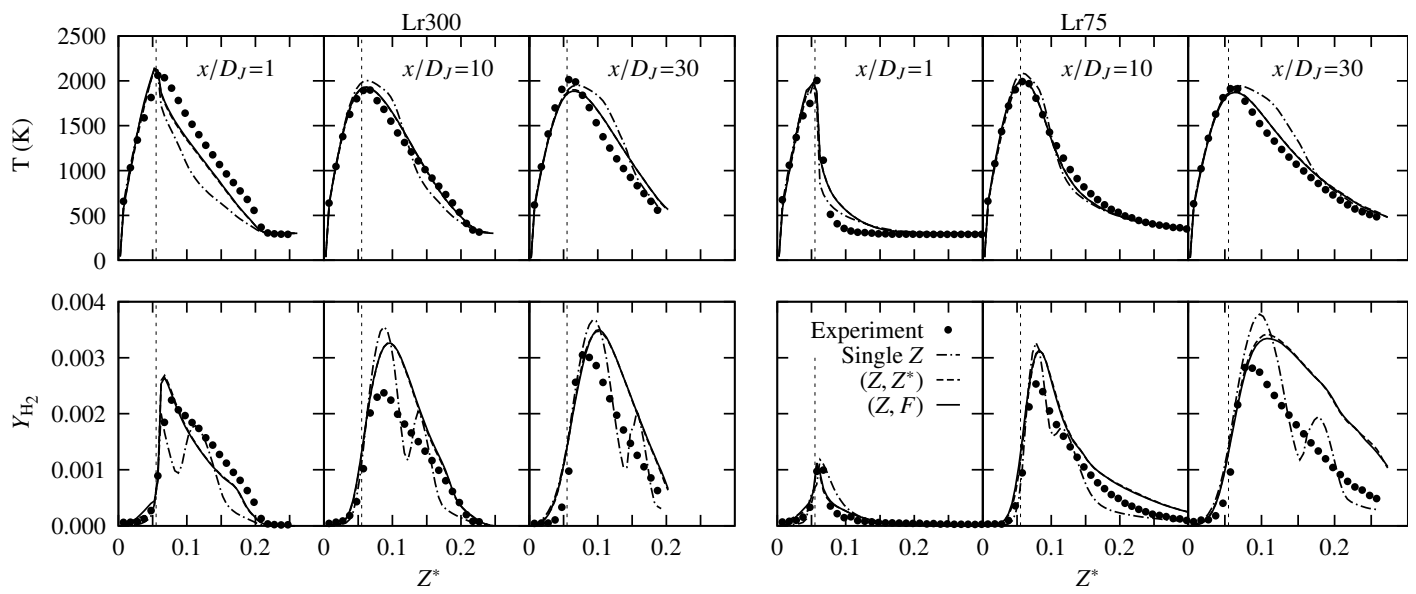

Figure 3: Conditional temperature (top row) and $Y_{\mathrm{H}_{2}}$ (bottom row) profiles at three axial locations downstream of the nozzle. Profiles on the left correspond to the homogeneous $(\operatorname{Lr} 300)$ case and profiles on the right correspond to the inhomogeneous (Lr75) case. The dashed vertical lines indicate the stoichiometric mixture fraction. The LES results correspond to statistics of the resolved quantities.

the two mixture fraction models and the experiment is a result of a slight misalignment of the central tube that resulted in asymmetrical measurements near the exit plane for the Lr300 case [13]. The larger underprediction of temperature for the single mixture fraction model indicates that the slight inhomogeneity in the inlet condition does have an impact on the flame structure in the near-nozzle region. Moving further downstream, the $\left(Z, Z^{*}\right)$ and $(Z, F)$ models remain essentially identical and continue to accurately predict temperature. In contrast, the single mixture fraction model predicts a spurious shoulder in the temperature profile on the rich side of the flame.

The trends observed when comparing the conditional profiles of $Y_{\mathrm{H}_{2}}$ in the bottom left row of Fig. 3 are similar to those for temperature but more pronounced. The larger differences are expected because the values of $Y_{\mathrm{H}_{2}}$ in the flamelet solutions are more sensitive to the boundary conditions. Again, no differences are observed between the $\left(Z, Z^{*}\right)$ and $(Z, F)$ models, and the agreement with the experimental data is good apart from a slight overprediction of $Y_{\mathrm{H}_{2}}$ on the rich side of the flame at the downstream locations. The qualitative agreement using the single mixture fraction model is substantially worse, for a spurious second peak in $Y_{\mathrm{H}_{2}}$ is predicted.

The qualitative discrepancies in the temperature and $Y_{\mathrm{H}_{2}}$ profiles observed for the single mixture fraction model are a result of the nonphysical boundary condition that is used for the fuel side of the flamelet equation in these cases. The boundary condition used implies that a cold, pure fuel stream exists at $Z=Z^{*}=1$, so the flamelet profiles for $T$ and $C$ are $\Lambda$-shaped, peaking near the stochiometric mixture fraction and terminating at $Z^{*}=1$. In the Sydney flame, the cold fuel boundary occurs at a variable, but less than unity, value of $Z^{*}$, so the flame $T$ and $C$ profiles are again $\Lambda$-shaped with peaks near the stiochiometric mixture fraction but terminate at $Z^{*} \sim 0.25$. As a result, the rich side of the flame profiles cross over and access thermochemical state information from unstable branch flamelet solutions, which have elevated levels of $\mathrm{H}_{2}$. This is purely an artifact of the nonphysical flamelet boundary condition and results in the second $Y_{\mathrm{H}_{2}}$ peak. Adding the second mixture fraction provides information to determine the appropriate flamelet equation boundary condition and alleviates this issue.

LES predictions of temperature and $Y_{\mathrm{H}_{2}}$ for the Lr75 case are compared to the experimental data on the right side of Fig. 3 . All models make similar predictions at $x / D_{J}=1$ but fail to predict the near-vertical temperature profile at stoichiometric mixture fraction that was observed experimentally. This behavior is a result of stratified-premixed combustion near the nozzle 
and is discussed in the next subsection. Both two mixture fraction models correctly predict temperature at the downstream locations, but the single mixture fraction model again predicts a spurious shoulder in the temperature profile. Similarly to the Lr300 case, the $(Z, F)$ and $\left(Z, Z^{*}\right)$ models qualitatively predict the trends in $\mathrm{H}_{2}$ formation, although the amount of $\mathrm{H}_{2}$ produced is overpredicted at $x / D_{J}=30$, and the single mixture fraction model predicts nonphysical second peaks in $Y_{\mathrm{H}_{2}}$ at $x / D_{J}=10$ and 30 .

Overall, the results for both cases indicate that two mixture fraction models are essential to even qualitatively predict the flame structure. For the Lr75 case, this is expected based on the large variation in $F$ across the flame. However, the trend also holds for the Lr300 case. This is because, even though the mean profiles of $F$ are nearly uniform, there are still temporal variations of $F$ near the nozzle, and a pure fuel boundary condition is used for the single mixture fraction model to ensure that $Z \leq 1$. This mismatch with the physically appropriate boundary condition results in inaccurate flame structure predictions. While the $\left(Z, Z^{*}\right)$ and $(Z, F)$ models have been shown to be equally accurate, the $(Z, F)$ model is more computationally expensive and less robust because an additional scalar transport equation with a modeled source term must be solved.

\subsection{Temperature scatter plots}

The differences in flame structure between the two cases can be examined in more detail through scatter plots of the instantaneous temperature conditioned on $Z^{*}$. These are shown in Fig. 4 for two axial locations in flames Lr300 and Lr75. For the Lr75 case at $x / D_{J}=1$, essentially all experimental data points on the rich side of the flame remain at $300 \mathrm{~K}$, corresponding to cold mixing between fuel and air. Near the stoichiometric mixture fraction, there is a steep rise in temperature, which is indicative of a propagating stratified-premixed combustion front. This occurs because the inhomogeneous inlet conditions place flammable, near-stoichiometric mixtures adjacent to the pilot.

The LES results using the $\left(Z, Z^{*}\right)$ model reproduce similar features, but, for the Lr75 case at $x / D_{J}=1$, a larger portion of the samples above the rich flammability limit have elevated temperatures, indicating diffusion-dominated, nonpremixed combustion. Note that the two mixture fraction models, although modified to account for the effects of inhomogeneity, are fundamentally based on an assumption of nonpremixed combustion and cannot predict premixed flame propagation. Therefore,

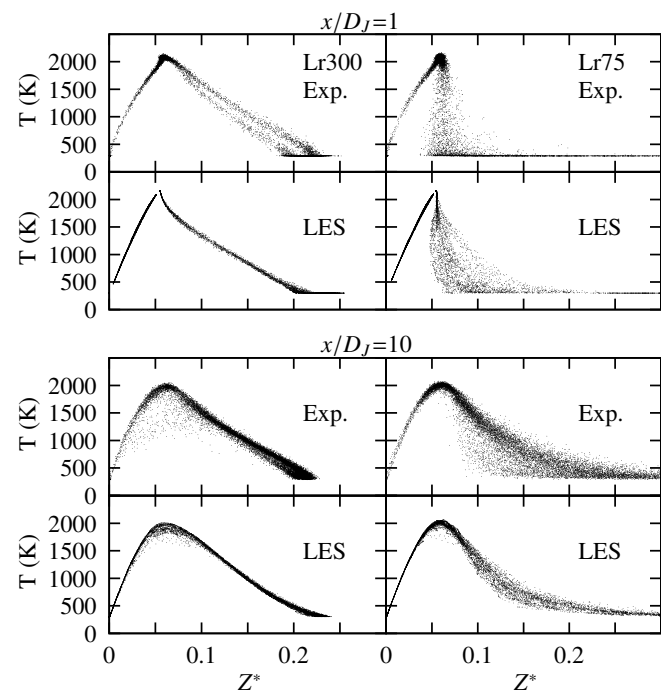

Figure 4: Instantaneous temperature scatter data conditioned on $Z^{*}$ at $x / D_{J}=1$ (top) and 10 (bottom). Data for the Lr300 and Lr75 cases are plotted in the left and right columns, respectively. The LES data correspond to the resolved temperature and were generated using the $\left(Z, Z^{*}\right)$ model. 
the difference between the model and experiment indicates that the near-nozzle region of the inhomogeneous flame is dominated by premixed or stratified combustion.

The experimental and LES scatter plots for the Lr300 case and the downstream region of the Lr75 case follow profiles characteristic of diffusion-dominated, nonpremixed combustion, but have minor differences. The two bands on the rich side of the $\operatorname{Lr} 300$ experimental data at $x / D_{J}=1$ and the wider scatter at $x / D_{J}=10$ result from asymmetry between the $+r$ and $-r$ sides of the flame. This accounts for the difference between the LES predictions and the mean experimental data that was previously described. Additionally, the presence of samples in the experimental data with significantly lower than equilibrium temperature near the stoichiometric mixture fraction indicates that there is some local extinction for the Lr300 case at $x / D_{J}=10$. Extinction is not observed for the Lr75 case because it is further from blowoff as a result of the stability benefit due to inhomogeneous mixing. The LES results do not show evidence of extinction, which steady flamelet models are known to underpredict [29], for either case.

\section{Conclusions}

A two mixture fraction flamelet model for simulation of turbulent flames with inhomogeneous inlets has been presented. The flamelet solution library associated with this model can be parametrized using either the two mixture fractions or one mixture fraction and a nonconserved scalar quantity termed the fuel premixing fraction. Using the Sydney burner with inhomogeneous inlets as a test case, it has been shown that single mixture fraction models are inadequate to accurately capture the flame structure of a combustion system with inhomogeneous inlets, but the two mixture fraction models can accurately predict the flame structure. The choice of parametrization for the two mixture fraction model has minimal impact on the results, but the $\left(Z, Z^{*}\right)$ approach is preferred because it avoids the complexity and consistency issues that result from solving the nonconserved scalar equation for $F$. The results of the simulations using the two mixture fraction model reinforce experimental observations about the effect of inhomogeneous inlets on flame structure and the role of stratified-premixed combustion in the near-nozzle region for the inhomogeneous case. The latter effect is not included in the current nonpremixed model but will be added in future model iterations.

\section{Acknowledgements}

B.A.P. gratefully acknowledges support from the National Science Foundation Graduate Research Fellowship Program under Grant DGE 1148900. A.R.M. is supported by the Australian Research Council.

\section{References}

[1] H. Pitsch, Ann. Rev. Fluid Mech. 38 (2006) 453-482.

[2] C.Y. Choi, R.D. Reitz, Fuel 78 (1999) 1303-1317.

[3] B.B. Dally, A.N. Karpetis, R.S. Barlow, Proc. Combust. Inst. 29 (2002) 1147-1154.

[4] C. Hasse, N. Peters, Proc. Combust. Inst. 30 (2005) 2755-2762.

[5] E.M. Doran, H. Pitsch, D.J. Cook, Proc. Combust. Inst. 34 (2013) 1317-1324. 
[6] M. Ihme, Y.C. See, Proc. Combust. Inst. 33 (2011) 1309-1317.

[7] M. Ihme, J. Zhang, G. He, B. Dally, Flow Turb. Combust. 89 (2012) 449-464.

[8] M. Stöhr, C.M. Arndt, W. Meier, Proc. Combust. Inst. 35 (2015) 3327-3335.

[9] L.Y.M. Gicquel, G. Staffelbach, T. Poinsot, Prog. Energy Combust. Sci. 38 (2012) 782-817.

[10] S. Meares, A.R. Masri, Combust. Flame 161 (2014) 484-495.

[11] S. Meares, V. Prasad, G. Magnotti, R.S. Barlow, A.R. Masri, Proc. Combust. Inst. 35 (2015) 1477-1484.

[12] A.R. Masri, R.W. Bilger, Proc. Combust. Inst. 21 (1986) 1511-1520.

[13] R.S. Barlow, S. Meares, G. Magnotti, H. Cutcher, A.R. Masri, Combust. Flame 162 (2015) 3516-3540.

[14] R.S. Barlow, J.H. Frank, Proc. Combust. Inst. 27 (1998) 1087-1095.

[15] N. Peters, Prog. Energy Combust. Sci. 10 (1984) 319-339.

[16] N. Peters, Turbulent Combustion, Cambridge University Press, Cambridge, U.K., 2000.

[17] H. Pitsch, N. Peters, Combust. Flame. 114 (1998) 26-40.

[18] C. Pierce, P. Moin, J. Fluid Mech. 504 (2004) 73-97.

[19] M. Ihme, H. Pitsch, Phys. Fluids 20 (2008) 055110.

[20] M. Germano, U. Piomelli, P. Moin, W. Cabot, Phys. Fluids A 3 (1991) 1760-1765.

[21] D.K. Lilly, Phys. Fluids A 4 (1992) 633-635.

[22] E.M. Doran, A Multi-Dimensional Flamelet Model for Ignition in Multi-Feed Combustion Systems, PhD Thesis, Stanford University, 2011.

[23] Y. Chen, M. Ihme, Combust. Flame 160 (2013) 2896-2910.

[24] V. Raman, H. Pitsch, Proc. Combust. Inst. 31 (2007) 1711-1719.

[25] O. Desjardins, G. Blanquart, G. Balarac, H. Pitsch, J. Comp. Phys. 227 (2008) 7125-7159.

[26] X. Liu, S. Osher, T. Chan, J. Comp. Phys. 115 (1994) 200-212.

[27] H. Pitsch, FlameMaster. A C++ computer program for OD combustion and 1D laminar flame calculations.

[28] G.P. Smith, D.M. Golden, M. Frenklach, GRI-Mech 3.0, available at http://www.me.berkeley.edu/gri_mech.

[29] M. Ihme, C.M. Cha, H. Pitsch, Proc. Combust. Inst. 30 (2005) 793-800. 


\section{List of Figures}

a 1 Two-dimensional state space for mixing between fuel in the jet, air in the jet (Air-J), and air in the coflow $\left(\right.$ Air-C) parametrized by $\left(Z, Z^{*}\right)$ and $(Z, F)$. . . . . . . . . . . . . . . . . . . . . . . 4

व 2 Experimental measurements (points) and $(Z, F)$ model predictions (lines) for the mean radial mixture fraction profiles at $x / D_{J}=1,10$, and 30 are shown in the top row. The arrows indicate increasing distance downstream.

The overlays show predictions of the instantaneous mixture fraction distribution at the nozzle exit plane from

the simulations of the mixing tube. The conditional mean value of the fuel premixing fraction $(F)$ at the same

three axial locations is plotted in the bottom row. The LES results correspond to statistics of the resolved quantities. . . . . . . . . . . . . . . . . . . . . . . . . . 7

3 Conditional temperature (top row) and $Y_{\mathrm{H}_{2}}$ (bottom row) profiles at three axial locations downstream of the nozzle. Profiles on the left correspond to the homogeneous (Lr300) case and profiles on the right correspond

to the inhomogeneous (Lr75) case. The dashed vertical lines indicate the stoichiometric mixture fraction. The LES results correspond to statistics of the resolved quantities. . . . . . . . . . . . . . . . . . . . 8

a 4 Instantaneous temperature scatter data conditioned on $Z^{*}$ at $x / D_{J}=1$ (top) and 10 (bottom). Data for the Lr300 and Lr75 cases are plotted in the left and right columns, respectively. The LES data correspond to the resolved 